Mario R. Eden, Marianthi Ierapetritou and Gavin P. Towler (Editors) Proceedings of the $13^{\text {th }}$ International Symposium on Process Systems Engineering - PSE 2018

July 1-5, 2018, San Diego, California, USA (C) 2018 Elsevier B.V. All rights reserved.

\title{
Multi-stage integrated electricity procurement and production scheduling
}

\author{
Egidio Leo $^{a^{*}}$ and Sebastian Engell ${ }^{a}$ \\ ${ }^{a}$ Process Dynamics and Operations Group, Department of Biochemical and Chemical \\ Engineering, Technische Universität Dortmund, EmilFigge-Str.70, 44221 Dortmund, \\ Germany \\ egidio.leo@tu-dortmund.de
}

\begin{abstract}
For optimal operation of power-intensive plants, the challenge addressed in this work is to determine simultaneously the optimal electricity procurement and the production scheduling. Electricity procurement decisions involve load commitment decisions, that mean the consumers have to commit themselves to the amount of electricity they are going to purchase before the actual electricity demand is reviled. A major challenge lies in the uncertainty. For this purpose a stochastic multi-stage mixed-integer linear programming model is developed that considers product demand and equipment breakdowns to determine the integrated electricity procurement and production scheduling. The proposed model is applied to a continuous power intensive plant and the results show the improvement achieved by solving the stochastic model.
\end{abstract}

Keywords: Electricity procurement, Production scheduling, Stochastic programming, Demand Side Management, load deviations.

\section{Introduction}

The increasing share of renewable energies, time-sensitive electricity prices and the flexibility of the production resources render industrial Demand-Side Response (iDSR) a promising strategy to maintain profitability for the process industry (Merkert et al., 2014). Besides exploiting production flexibility to take advantage of time sensitive electricity prices, a challenge of iDSR is the integration of electricity procurement and production scheduling to avoid the risk of having competing optimization solutions resulting in no improvements. This means the introduction of electricity purchasing decisions into the scheduling problem. For large electricity consumers, the electricity purchase sources include different power contracts:

- Long-term contract (base load) - constant price, constant amount of electricity delivered over time and horizon of at least 1 year;

- Time-of-Use contract (TOU) - price levels consisting of a time-dependent component and an amount-dependent component; TOU contract covers a period of time between 1 week and 3 months;

- Day-ahead market (spot market) - hourly-varying prices.

The day-head spot market is characterized by hourly varying electricity prices, according to the regional fluctuations of electricity availability. The day-ahead electricity price is assumed to be known $24 \mathrm{~h}$ ahead. Apart from the volatility of the electricity price, a main feature of the day-ahead spot market is the load deviation problem and its related deviation penalties. In fact, for large consumers, the electricity suppliers impose a daily day-ahead hourly electricity commitment and in case the actual 
consumption deviates from the pre-agreed values, financial penalties, which are often in the same range as the net electricity cost, are incurred. In (Hadera et al., 2015), the authors take into account these multiple electricity contracts and the load deviation problem to determine the optimal production schedule. However, the contract-related decisions (e.g. the load commitments) are assumed as given and not optimized. In this work, we address the challenge of determining simultaneously the optimal electricity purchase strategy and the production scheduling including the load commitments. Load commitment means the commitment of the consumers to the amount of electricity that they are going to purchase and use during a certain period of time. Depending on the time-scale, we can distinguish between the TOU commitment, which covers up to 3 months, and the daily day-ahead hourly commitment. Since decisions regarding the commitment to power contracts have to be made before the actual electricity demand is known for the time horizon of interest, it is crucial to account for uncertainties in the decision-making process. A recent work in this direction (Zhang et al., 2016) integrates production scheduling and TOU-contract decisions applying two-stage stochastic programming (Birge and Louveaux, 2011) to model price and demand uncertainty. However, this work does not consider the day-ahead electricity commitment and its related load deviation problem. To include the day-ahead commitment and the load deviation problem, we propose a three-stage stochastic programming approach. The first-stage variables represent the electricity commitment decisions related to the TOU power contracts. The second stage faces demand uncertainty and for each demand scenario the second-stage variables represent the day-ahead electricity commitment. The third stage is used to take into account plant capacity uncertainty and third-stage variables for each scenario are the plant operating decisions (production levels, inventories etc.) and the deviations of the power demand from the day-ahead commitment. A similar approach has not been considered in this context before. In Section 2, the uncertainty modelling strategy and the proposed three-stage MILP are presented. Section 3 applies the proposed framework to a power-intensive process and the results are summarized in terms of the Value of the Stochastic Solution (VSS). The main outcomes from the results are discussed in Section 4 before providing some final conclusion in Section 5.

\section{Problem statement}

\subsection{Uncertainty modeling strategy}

To integrate electricity procurement and production scheduling, we proposed a multistage stochastic programming approach (Birge and Louveaux, 2011). In stochastic programming, uncertainty is represented by discrete scenarios, and decisions are made at different stages, defined by the realization of the uncertainty. In the proposed approach we consider two sources of uncertainty: product demand faced by the second stage and equipment breakdown faced by the third stage. For each source of uncertainty we consider three different levels-low, medium, and high with the same expected value. The low, medium and high uncertainty levels represent respectively a variation of 5,10 and 20 percent of the product demand and 10,30 and 40 percent reduction of the maximum plant production capacity. The production capacity reduction is modeled by 8 breakdown scenarios: In scenario 1 no breakdown occurs, whereas in Schemes 2-8 the breakdown occur in periods $1-4, \ldots, 22-24$, respectively. The probabilities are $50 \%$ for scenario 1 and (50/7)\% for scenario 2-8. 


\subsection{Plant model}

To demonstrate the main features of the proposed approach, we apply it to a continuousproduction plant. The plant scheduling model follows what proposed in (Zhang et al., 2016). Note that in the proposed mathematical formulation first-stage variables have no scenario subscript, second stage variables have the scenario subscript $s$ and the thirdstage variables have a double scenario subscript $s, \bar{s}$. The plant produces two products $i$ $(P 1$ and $P 2)$ and it can operate in three different modes $m$ : off, startup, and on. The possible mode transitions are off to startup, startup to on, and on to off and they can happen only after fixed stay times (off: 8 h, startup:2 h, on:6 h) (Eq.6). The transitions between the modes of operation and the relations with the active modes are modelled by Eq.(7). The binary variable (Eq.(4)) is 1 if mode $m$ is selected in time period $t$ of the horizon $T$. For each mode the operating conditions are expressed as a convex combination of the extreme points $v_{m i}$ of the feasible region of operation (Eq.(2)). Eq.(1)-(2) define the hourly production level $\overline{P D}_{m i t s \bar{s}}$ for each product $i$ and operating mode $m$ and the aggregated production $P D_{i t s \bar{s}}$.

$$
\begin{array}{lr}
P D_{i t s \bar{s}}=\sum_{m} \overline{P D}_{m i t s \bar{s}} & \forall i, t \in T, s, \bar{s} \\
\overline{P D}_{m i t s \bar{s}}=\sum_{m} \lambda_{m i t s \bar{s}} * v_{m i} & \forall i, t \in T, s, \bar{s} \\
\sum_{m}^{m} \lambda_{m i t s \bar{s}}=y_{m t} & \forall i, t \in T, s, \bar{s} \\
\sum_{m}^{m} y_{m t}=1 & \forall t \in T \\
E U_{i t s \bar{s}}=\sum_{m}\left(\delta_{m} * y_{m t}+\sum_{i} \gamma_{m i} * \overline{P D}_{m i t s \bar{s}}\right) & \forall i, t \in T, s, \bar{s} \\
\theta_{m m \prime} & \forall\left(m, m^{\prime}\right) \in M, t \in T, s, \\
\sum_{k=1}^{k} z_{m m^{\prime} t-k} \leq y_{m \prime t} &
\end{array}
$$

Eq. (8) defines the inventory level $I V_{i t s \bar{s}}$ at time $t$ as the sum of the inventory level at time period $t-1$ and the production at time $t P D_{i t s \bar{s}}$ minus the amount of products sold $S L_{i t s \bar{s}}$ and the amount of products wasted $P W_{i t s \bar{s}}$ at time period $t$. Note that all these quantities are defined for each scenario of the second and third stage. Eq.(9) sets upper and lower bound of the inventory levels and Eq. (10) satisfies the demand of product $i$ as the sum of the amount of product $i$ sold $S L_{i t s \bar{s}}$ and the amount of product $i$ purchased from other sources $P C_{i t s \bar{s}}$.

$$
\begin{array}{ll}
I V_{i t s \bar{s}}=I V_{i, t-1, s \bar{s}}+P D_{i t s \bar{s}}-S L_{i t s \bar{s}}-P W_{i t s \bar{s}} & \forall i, t \in T, s, \bar{s} \\
I V_{i t}^{\text {min }} \leq I V_{i t s \bar{s}} \leq I V_{i t}^{\max } & \forall i, t \in T, s, \bar{s} \\
S L_{i t s \bar{s}}+P C_{i t s \bar{s}}=D_{i t s} & \forall i, t \in T, s, \bar{s}
\end{array}
$$

Eq. (11) - (14) provides the initial and final condition of the plant in terms of inventory levels and active operating mode.

$$
\begin{array}{lc}
I V_{i, 0, s, \bar{s}}=I V_{i}^{\text {initial }} & \forall i, s, \bar{s} \\
y_{m, 0}=y_{m}^{\text {initial }} & \forall m \\
I V_{i, t} \text { final }_{, s, \bar{s}} \geq I V_{i}^{\text {final }} & \forall i, s, \bar{s} \\
z_{m m \prime t}=z_{m m \prime t}^{\text {ini }} & \forall\left(m, m^{\prime}\right) \in M, s, \bar{s},-\theta^{\text {max }}+1 \leq t \leq-1
\end{array}
$$




\subsection{Power contract model}

Eq.(15)-(21) define the amount of electricity $\widehat{E C}_{c}$ purchased from TOU contract $c . B_{c}$ is the set of blocks of contract $c$, and $x_{c b}$ is a Boolean variable that is true if block $b$ of contract $c$ is chosen. $\widehat{E C}_{c, b}^{\max }$ is the amount of electricity that one has to purchase in block $b$ before reaching the next block; $\beta_{c b}$ is the quantity dependent price. Eq.(22) models the electricity commitments of the plant: $E C_{c t}$ represents the amount of energy the consumer purchases from the TOU contract and $E S_{t s}$ is the day-ahead electricity commitment. According to the timing of the decisions, TOU commitment decisions are first-stage variables and day-ahead commitment decisions are second-stage variables. Eq.(23) defines the over- $\left(\Delta e_{t s \bar{s}}^{+}\right)$and under-consumptions $\left(\Delta e_{t s \bar{s}}^{-}\right)$(third stage variables) to compute the penalty costs.

$$
\begin{array}{lc}
\widehat{E C}_{c}=\sum_{t} E C_{c t} & \forall c \\
\widehat{E C}_{c}=\sum_{b} \widetilde{E C}_{c b} & \forall c \\
B C_{c}=\sum_{b} \beta_{c b} \widetilde{E C}_{c b} & \forall c \\
\widehat{E C}_{c, b-1} \max _{c b} \leq \widetilde{E C}_{c b} & \forall c, b \in B_{c}, b>1 \\
\widetilde{E C}_{c b} \leq \widetilde{E C}_{c, b}^{\max } x_{c b} & \forall c, b \in B_{c} \\
\sum_{c b} x_{c b}=1 & \forall c \\
E C_{c t}=\overline{E C}_{c p} & \forall c, p \in P_{c}, t \in T_{c p} \\
Q_{t s}=E C_{c t}+E S_{t s} & \forall t \in T, s \\
E U_{t s \bar{s}}-Q_{t s}=\Delta e_{t s \bar{s}}^{+}-\Delta e_{t s \bar{s}}^{-} & \forall t \in T, s, \bar{s}
\end{array}
$$

\subsection{Objective function}

The model minimizes the total expected operating costs, $z$, defined in Eq.(24) as the cost of purchasing energy from the TOU contract, the expected first-stage cost of purchasing electricity from the spot market and the expected second-stage cost of deviation penalties and purchasing products.

$$
\begin{aligned}
z=\sum_{c} B C_{c}+\sum_{t} & \sum_{c} p_{c t}^{T O U} E C_{c t} \\
& +\sum_{s} \varphi_{s} \sum_{t}\left(p_{t}^{\text {day-ahead }} E S_{s t}\right. \\
& \left.+\sum_{\bar{s}} \varphi_{\bar{s}}\left(p_{t}^{+} \Delta e_{t s \bar{s}}^{+}+p_{t}^{-} \Delta e_{t s \bar{s}}^{-}+\sum_{i} p_{i} P C_{i t s \bar{s}}\right)\right)
\end{aligned}
$$

where $p_{c t}^{T O U}, p_{t}^{\text {day-ahead }}, p_{t}^{+}, p_{t}^{-}, p_{i}$ represent respectively the TOU electricity price, the day-ahead electricity price, the penalty cost for over and under consumption and the product purchasing price; $\varphi_{s}, \varphi_{s \bar{s}}$ denote the probability of scenario $S$ and $\bar{s}$.

\section{Results}

To measure the improvement that can be achieved by solving the stochastic model we compute the value of the stochastic solution (VSS). The VSS (Eq.(25)) and the relative $\overline{V S S}$ (Eq.(26)) show the impact of the uncertainty on the first-stage variables: 


$$
V S S=z_{\text {det }}^{*}-z_{\text {stoc }}^{*}
$$

$$
\overline{V S S}=\frac{z_{\text {det }}^{*}-z_{\text {stoc }}^{*}}{z_{\text {det }}^{*}}
$$

Where $z_{\text {stoc }}^{*}$ is the optimal solution of the stochastic problem and $z_{\text {det }}^{*}$ is the optimal solution of the stochastic problem with first-stage (and second stage for $V S S_{2}$ ) variables fixed to the optimal deterministic solution. Table 1 shows that the VSS can be quite significant and that it grows with the uncertainty level. The VSS clearly shows the benefit of adopting the stochastic model instead of the deterministic one. Each model has up to approximately 19,000 continuous variables, 212 binary variables, and 11,000 constraints. All models were solved to zero integrality gap in less than $20 \mathrm{~s}$ applying the solver CPLEX 12.6.3.0.

Table 1 Value of the stochastic solution for the proposed 3-stage stochastic model

\begin{tabular}{llcccc}
\hline $\begin{array}{l}\text { Demand } \\
\text { Uncertainty level }\end{array}$ & $\begin{array}{l}\text { Breakdown } \\
\text { uncertainty level }\end{array}$ & $\boldsymbol{V S S _ { \mathbf { 1 } }}$ & $\overline{\boldsymbol{V S S}}_{\mathbf{1}}$ & $\boldsymbol{V S S}_{\mathbf{2}}$ & $\overline{\boldsymbol{V S S}}_{\mathbf{2}}$ \\
\hline Low & Low & 105 & $4.3 \%$ & 144 & $5.8 \%$ \\
Low & Medium & 140.4 & $5.3 \%$ & 168.5 & $6.3 \%$ \\
Low & High & 130.3 & $4.7 \%$ & 157.8 & $6.0 \%$ \\
Medium & Low & 158.7 & $6.3 \%$ & 229 & $8.9 \%$ \\
Medium & Medium & 194.5 & $7.1 \%$ & 242.4 & $8.8 \%$ \\
Medium & High & 185.8 & $6.6 \%$ & 230.1 & $8.0 \%$ \\
High & Low & 222.8 & $8.4 \%$ & 338.3 & $12.2 \%$ \\
High & Medium & 249 & $8.7 \%$ & 367.6 & $12.4 \%$ \\
High & High & 245.2 & $8.3 \%$ & 348.8 & $11.4 \%$ \\
\hline
\end{tabular}

\section{Discussion}

Fig. 1 shows the electricity purchase profiles obtained for a medium uncertainty level solving the proposed 3-stage stochastic model (Fig.1 a) and 2-stage stochastic model (Fig.1 b) proposed in (Zhang et al., 2016), where the day-ahead electricity commitment and its related deviation problem are neglected. In the 2SSP model the uncertainty source is the product demand as in the second stage of the proposed 3SSP approach. Note that both approaches model the TOU contract decisions as first-stage variables. The base load profiles are supposed given and therefore not optimize since base load contract decisions have to be made before the time horizon of interest. The shown dayhead electricity purchase profiles are the expected value computed over all scenarios. In the 3SSP solution, the plant operates longer in order to accommodate for the highdemand and breakdown scenario providing more flexibility for load shifting. Accounting for breakdown scenarios means purchasing more electricity (when convenient) in order to accommodate production capacity reduction. This renders the TOU contract (according to the electricity prices) more convenient than the day-ahead market and therefore the 3SSP solution purchase a higher amount of electricity from the TOU contract. It is remarkable how different the optimal electricity purchase strategy could be considering the day-ahead commitment and the load deviation problem.

\section{Conclusion}

This work addresses the integrated electricity procurement and production scheduling for continuous power-intensive processes. A Three-stage stochastic programming is proposed to model electricity commitments taking into account product demand and equipment breakdown uncertainty. The application of the proposed approach to a power intensive plant shows the benefit of the stochastic model. Acknowledgements- The 
financial support from the Marie Sklodowska-Curie Horizon2020 project "PRONTO" (Contract No: 675215) is gratefully acknowledged.

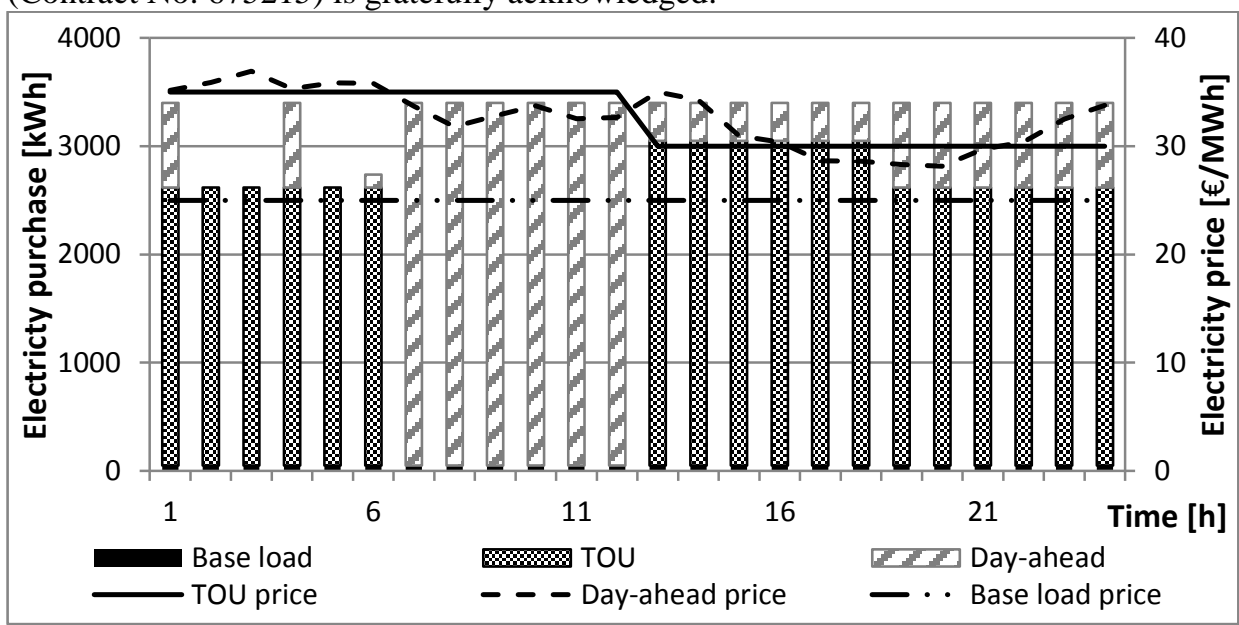

(a)

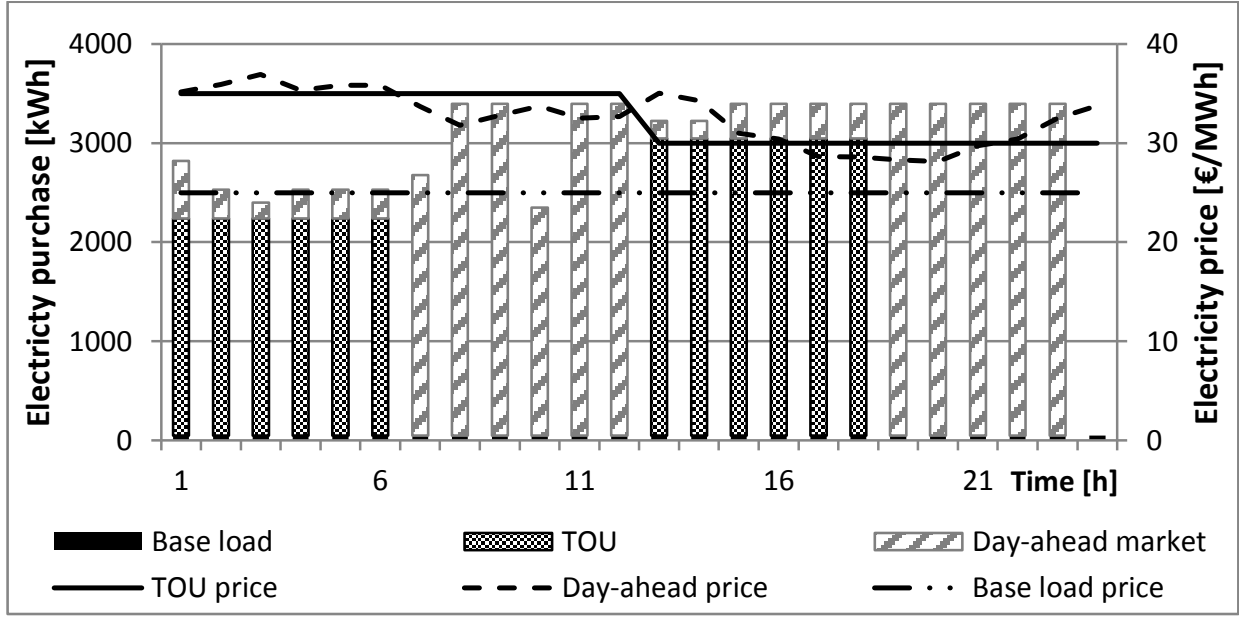

(b)

Figure 1 Electricity profiles obtained solving the 3-stage (a) and 2-stage (b) stochastic model

\section{References}

Q. Zhang, L. Jochen, L. Cremer, I.E. Grossmann, A. Sundaramoorthy and J.M. Pinto, 2016, Risk-based integrated production scheduling and electricity procurement for continuous power-intensive processes, Comput. Chem. Eng., 86, 90-105.

H.Hadera, I.Harjunkoski, G.Sand, I.E.Grossmann and S.Engell, 2015, Optimization of steel production scheduling with complex time-sensitive electricity cost, Comput. Chem. Eng., 76.

JR. Birge, F. Louveaux.2011, Introduction to stochastic programming, Springer Science+Business

L.Merkert, I.Harjunkoski, A.Isaksson, S.Säynevirta, A. Saarela and G. Sand, 2014, Scheduling and energy - industrial challenges and opportunities, Comput. Chem. Eng., 72, 183-198. 